\title{
LASER SPECTROSCOPY OF OPTICAL CENTERS IN BOROSILICOPHOSPHATE GLASS, COACTIVATED BY ERBIUM AND YTTERBIUM IONS
}

\author{
M. V. Bogdanovich, ${ }^{1}$ A.I. Enzhievskiii, ${ }^{1 *}$ G. I. Ryabtsev, ${ }^{1}$ \\ V. V. Kuznetsova, ${ }^{2}$ V. V. Mashko, ${ }^{2}$ L. L. Teplyashin, ${ }^{2}$ \\ L. I. Burov ${ }^{3}$ A. G. Ryabtsev, ${ }^{3}$ M. A. Shchemelev, ${ }^{3}$ \\ and A. S. Kraskovskii ${ }^{4}$
}

UDC 535.37:621.373.826

Based on analysis of the laser emission spectra of borosilicophosphate glass, coactivated by $\mathrm{Er}^{3+}$ and $\mathrm{Yb}^{3+}$ ions, we have determined the structure of the Stark splitting of the luminescence band for the erbium ion in the $1.5 \mu \mathrm{m}$ region (the transition ${ }^{4} \mathrm{I}_{13 / 2} \rightarrow{ }^{4} \mathrm{I}_{15 / 2}$ ). In the wavelength interval $1532-1547 \mathrm{~nm}$, we identified 12 sets of lines belonging to different types of optical centers of predominantly cubic symmetry.

Key words: Er,Yb:borosilicophosphate glass, diode-pumped laser, optical center, Stark splitting.

Introduction. Recently the characteristics of glasses coactivated by different rare-earth ions such as $\mathrm{Yb}^{3+}$ and $\mathrm{Er}^{3+}$ have been intensively studied. This is connected primarily with the possibility of using such glasses as active media for diode-pumped lasers, lasing in the eye-safe spectral range $(\sim 1.5 \mu \mathrm{m})[1-3]$, and also for upconversion visualization of IR laser radiation fields [4].

It was shown previously [5] that in the lasing spectrum of a laser based on borosilicophosphate (BSP) glass coactivated by $\mathrm{Er}^{3+}$ and $\mathrm{Yb}^{3+}$, we observe two groups of narrow lines within the IR luminescence band, corresponding to the optical transitions ${ }^{4} I_{13 / 2} \rightarrow{ }^{4} I_{15 / 2}$ in the $\mathrm{Er}^{3+}$ ions. The number of lines, their spectral position, and also the time dependence of the intensity of the laser emission depend on the pump power. The total number of lines in the lasing spectrum for an erbium laser, for sufficiently high excitation power, substantially exceeds the maximum possible number of components for Stark splitting of the ${ }^{4} I_{15 / 2}$ level typical for $\mathrm{Er}^{3+}$ optical centers of the lowest symmetry $(8$ components). The presence of a large number of lines in the lasing spectrum for Er,Yb:BSP glass is due to the appearance of several types of optical centers, differing in the immediate environment of the rare earth ion, where each of them has its own Stark splitting structure.

In order to improve the characteristics of laser emitters based on borosilicophosphate glass, it is important to know the basic properties of the optical centers capable of having an effect on the power, spectral, and temporal parameters of the generated emission. This work has been devoted to a detailed study of the features of the Stark splitting structure for the ground state level ${ }^{4} I_{15 / 2}$ of the erbium ion for different types of optical centers in borosilicophosphate glass coactivated by $\mathrm{Er}^{3+}$ and $\mathrm{Yb}^{3+}$ ions. We used the intracavity laser spectroscopy method, in which the active medium itself acts as the object of investigation. We paid special attention to classification of the optical centers and study of when they appear under lasing conditions.

The experiment. The studied sample of Er,Yb:BSP glass, in the form of a rod of length $11 \mathrm{~mm}$ and diameter $3 \mathrm{~mm}$, was made at the A. M. Prokhorov Institute of General Physics (Russian Academy of Sciences). The properties of this glass are analogous to the properties of the samples used in $[6,7]$. The concentrations of $\mathrm{Er}^{3+}$ and $\mathrm{Yb}^{3+}$ ions were $5 \cdot 10^{19}$ and $4 \cdot 10^{21} \mathrm{~cm}^{-3}$. The Er,Yb:BSP glass was excited by emission from a high-power laser diode linear

*To whom correspondence should be addressed.

${ }^{1}$ B. I. Stepanov Institute of Physics, National Academy of Sciences of Belarus, 68 prosp. Nezavisimosti, Minsk 220072, Belarus. E-mail: ryabtsev@dragon.bas-net.by; ${ }^{2}$ Institute of Atomic and Molecular Physics, National Academy of Sciences of Belarus, Minsk; ${ }^{3}$ Belorussian State University, Minsk; ${ }^{4}$ Peleng OAO, Minsk, Belarus. Translated from Zhurnal Prikladnoi Spektroskopii, Vol. 73, No. 4, pp. 483-487, July-August, 2006. Original article submitted January 31, 2006. 


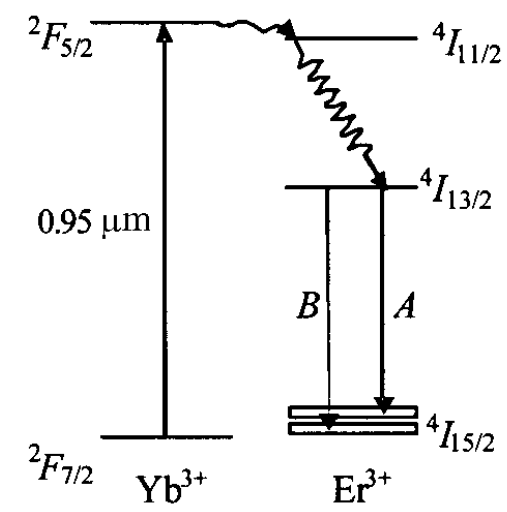

Fig. 1 Energy level diagram for $\mathrm{Er}^{3+}$ and $\mathrm{Yb}^{3+}$ ions in borosilicophosphate glasses.

array according to a transverse pumping scheme. We used two or four laser diode linear arrays, located along the lateral surface of the active element symmetrically relative to the longitudinal axis of the cavity. The maximum pump power when using two and four linear diode linear arrays was $100 \mathrm{~W}$ and $200 \mathrm{~W}$. The emission wavelength of the laser diode linear array $(\lambda \approx 0.95 \mu \mathrm{m})$ corresponded to the position of an intense absorption band for the $\mathrm{Yb}^{3+}$ ions, which are efficient sensitizers of $\mathrm{Er}^{3+}$ ions. The pulse duration for the pump emission was $\sim 5 \mathrm{msec}$.

The laser cavity was formed by two plane mirrors with reflection coefficients of $99 \%$ (opaque) and $90 \%$ (output mirror) at a lasing wavelength of $\lambda \approx 1.54 \mu \mathrm{m}$. The geometric length of the cavity was $75 \mathrm{~mm}$, intermode spacing was $0.014 \mathrm{~nm}$. Under free-lasing conditions, the duration of the output pulse was varied within the range 0.5-5.0 msec, depending on the pump power.

The luminescence and lasing spectra were recorded by an InGaAs CCD linear array, mounted at the output of a spectral diffraction camera. The lasing spectra were measured for different pump powers $(P=30,60,100$, and 200 W) and cavity losses. The losses were varied by small misadjustments of the cavity mirrors.

Results and discussion. The electronic level diagram, with an indication of radiative and nonradiative transitions, is shown in Fig. 1 for erbium and ytterbium ions in borosilicophosphate glasses. The first excited level ${ }^{4} I_{13 / 2}$ of the $\mathrm{Er}^{3+}$ ions is populated mainly by energy transfer from the $\mathrm{Yb}^{3+}$ ions, absorbing the pump emission in the transition ${ }^{2} F_{7 / 2} \rightarrow{ }^{2} F_{5 / 2}(\sim 0.95 \mu \mathrm{m})$. The ground state energy level ${ }^{4} I_{15 / 2}$ of the $\mathrm{Er}^{3+}$ ions in borosilicophosphate glasses consists of two groups of inhomogeneously broadened sublevels [5], to which correspond the maxima $A$ ( $\lambda \sim 1541.6$ $\mathrm{nm})$ and $\mathrm{B}(\lambda \sim 1534.9 \mathrm{~nm})$ of the luminescence band (Fig. 2a). Such a shape for the luminescence band is typical for most erbium glasses [8].

The lasing spectra for a laser based on Er,Yb:BSP glass for different excitation conditions and cavity losses are presented in Fig. 2b-r. Near the threshold pump power level of $30 \mathrm{~W}$, in the vicinity of the $B$ maximum, one lasing line appears (Fig. 2b). Increasing $P$ up to $100 \mathrm{~W}$ leads to an increase in the number of lines near the $B$ maximum and the appearance of an additional group of lines near the $A$ maximum (Fig. 2c and d). A change in the cavity losses for pump level $100 \mathrm{~W}$ has practically no effect on the spectral position of these two groups of lines (Fig. 2e-i). For an excitation level of $200 \mathrm{~W}$, within the entire luminescence band for Er,Yb:BSP glass, new groups of lasing lines arise (Fig. 2j-r). The spectral interval between adjacent lasing lines is significantly greater than the intermode spacing of the cavity, and consequently observation of a large number of lines cannot be connected with the mode structure of the cavity.

Results of analysis of the lasing spectra obtained, some of which are shown in Fig. 2, suggest that the appearance of different lines in the lasing spectra for Er,Yb:BSP glass is not accidental. Depending on the excitation conditions, in the laser emission we can pick out at least 12 sets of interconnected individual lines. Each such set is characterized by a certain number of lines: five or eight. All lines in a set appear as a single ensemble in one or several lasing spectra. It is important to note that a line with a certain spectral position belongs to only one set. For $P \leq 100 \mathrm{~W}$, we observe at least five sets of lines. Increasing the pump power up to $200 \mathrm{~W}$ leads to the appearance 

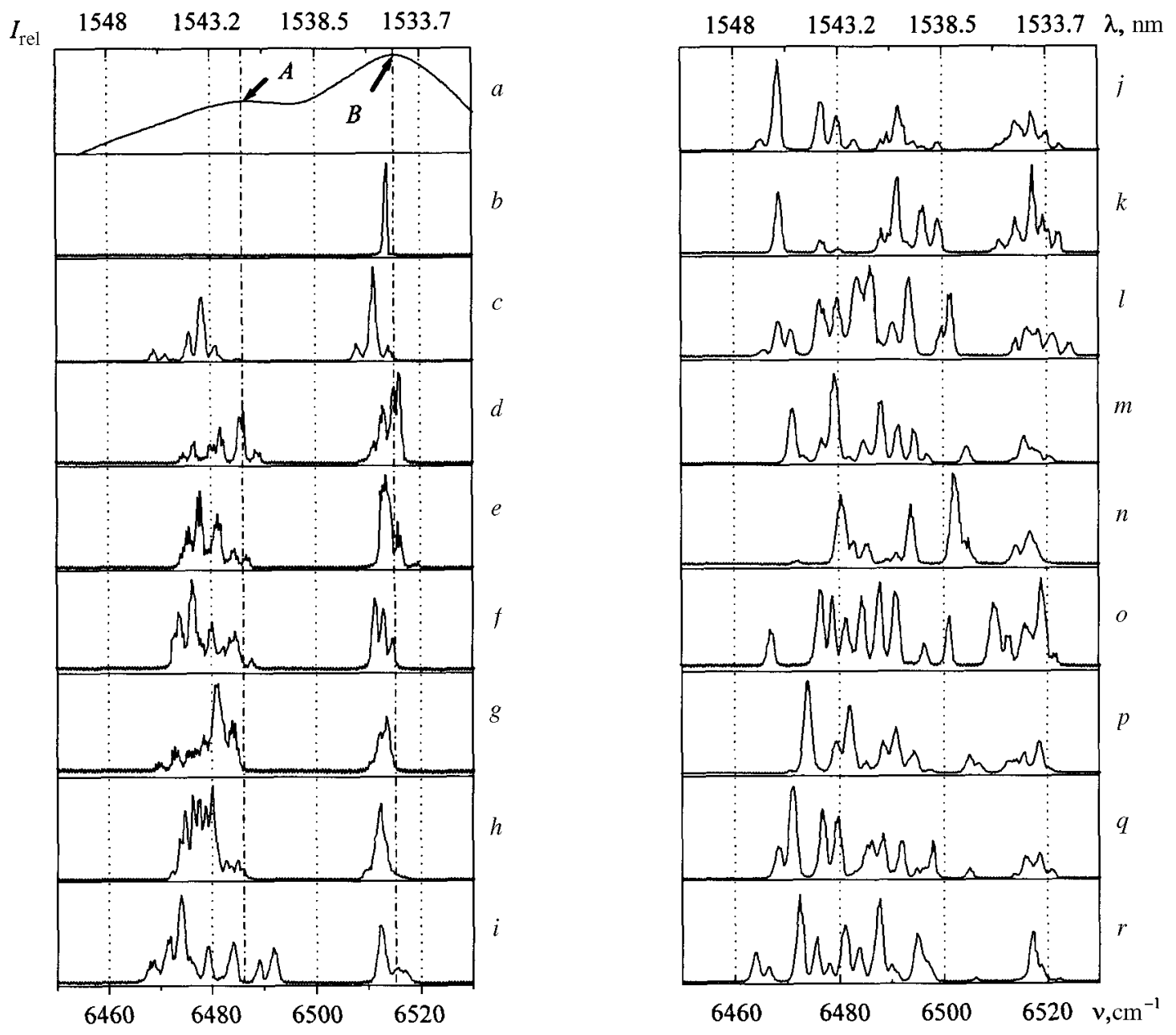

Fig. 2 Luminescence spectrum (a) and lasing spectra (b-r) for Er,Yb:BSP glass for different intracavity losses and pump levels: $W_{\text {pump }}=30 \mathrm{~W}(\mathrm{~b}), 60 \mathrm{~W}(\mathrm{c})$ $100 \mathrm{~W}(\mathrm{~d}-\mathrm{i})$, and $200 \mathrm{~W}(\mathrm{j}-\mathrm{r})$.

in the lasing spectra of at least seven more sets. The wavenumbers of the individual lines in the sets, which appear for excitation conditions $\mathrm{P} \leq 100 \mathrm{~W}$ and $P=200 \mathrm{~W}$, are presented in Table 1.

As shown in [5], the lasing lines for Er,Yb:BSP glass correspond to transitions from lower components of the Stark splitting of the level ${ }^{4} I_{13 / 2}$ to several components of the Stark splitting of the level ${ }^{4} I_{15 / 2}$. The maximum number of components of the Stark splitting of the level ${ }^{4} I_{15 / 2}$ for the $\mathrm{Er}^{3+}$ ion in an intramolecular electrostatic field of low symmetry is equal to eight [9]. Since in the laser emission of Er,Yb:BSP glass we observe spectral sets of five or eight related lines, we can hypothesize that during lasing under the given experimental conditions, at least 12 types of optical centers are involved that have different Stark splitting structures. The individual types of centers differ in the symmetry and strength of the electrostatic field created by the immediate environment, which affects the nature of the Stark splitting. The lasing lines for each set correspond to radiative transitions from one lower Stark component of the level ${ }^{4} I_{13 / 2}$ to several Stark components of the level ${ }^{4} I_{15 / 2}$ of the corresponding optical center. The positions of the Stark components of the splitting for the optical centers, obtained on the basis of the data in Table 1, are shown in Fig. 3 for the cases $P \leq 100 \mathrm{~W}$ and $P=200 \mathrm{~W}$. We took the values of the energies for the shortest wavelength lines as the zero reference points for the energy levels of the Stark components for each center.

As follows from Fig. 3a, for optical centers 1-5, typically the Stark components are combined into two groups with wavenumbers in the ranges $0-8 \mathrm{~cm}^{-1}$ and $25-45 \mathrm{~cm}^{-1}$. Such grouping of the Stark components for the 

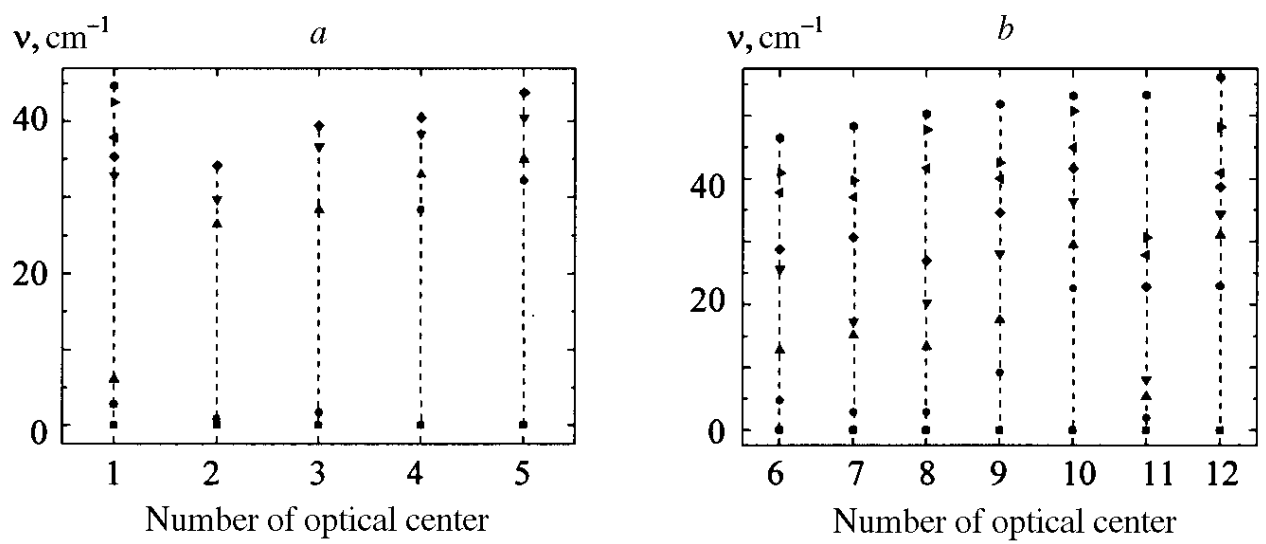

Fig. 3 Diagram of the Stark structure for the level ${ }^{4} I_{15 / 2}$ for $\mathrm{Er}^{3+}$ ions for different optical centers in borosilicophosphate glasses, appearing for pump power up to $100 \mathrm{~W}$ (a) and above $200 \mathrm{~W}$ (b).

TABLE 1 Wavenumbers for Individual Lines of the Sets Appearing for Excitation Levels up to $100 \mathrm{~W}$ and $200 \mathrm{~W}$

\begin{tabular}{|c|c|c|c|c|c|c|c|c|c|c|c||}
\hline \multicolumn{7}{|c|}{ Set (up to $100 \mathrm{~W}$ ) } & \multicolumn{8}{c|}{ Set (200 W) } \\
\hline 1 & 2 & 3 & 4 & 5 & 6 & 7 & 8 & 9 & 10 & 11 & 12 \\
\hline 6517.60 & 6515.93 & 6512.85 & 6512.30 & 6513.41 & 6521.29 & 6519.59 & 6518.48 & 6518.73 & 6517.33 & 6522.39 & 6524.65 \\
6514.82 & 6515.09 & 6511.18 & 6483.92 & 6481.18 & 6516.53 & 6516.78 & 6515.68 & 6509.52 & 6494.77 & 6520.44 & 6501.70 \\
6511.48 & 6489.47 & 6484.48 & 6479.22 & 6478.39 & 6508.43 & 6504.49 & 6505.08 & 6501.15 & 6487.82 & 6517.08 & 6493.60 \\
6484.65 & 6486.14 & 6476.18 & 6473.96 & 6472.86 & 6495.62 & 6502.29 & 6498.11 & 6490.60 & 6480.88 & 6514.28 & 6490.30 \\
6482.18 & 6481.71 & 6473.71 & 6471.76 & 6469.55 & 6492.54 & 6488.91 & 6491.44 & 6484.12 & 6475.64 & 6499.50 & 6485.93 \\
6479.70 & - & - & - & - & 6483.40 & 6482.52 & 6476.77 & 6478.69 & 6472.32 & 6494.48 & 6483.66 \\
6475.03 & - & - & - & - & 6480.34 & 6479.79 & 6470.69 & 6476.18 & 6466.55 & 6491.69 & 6476.47 \\
6472.83 & - & - & - & - & 6474.80 & 6471.21 & 6468.18 & 6466.79 & 6464.05 & 6469.02 & 6468.47 \\
\hline \hline
\end{tabular}

level ${ }^{4} I_{15 / 2}$ of the rare-earth ion is characteristic of crystal matrices in which the optical centers are located in positions of cubic symmetry, such as Er:YAG [10, 11], and also various other garnets activated by neodymium ions [12]. The total Stark splitting of the level ${ }^{4} I_{15 / 2}$ for optical centers in Er,Yb:BSP glass is $\sim 45 \mathrm{~cm}^{-1}$, which is an order of magnitude smaller than for crystals with cubic symmetry: for example, for Er:YAG, this quantity is $\sim 600 \mathrm{~cm}^{-1}[10]$. The small value of the Stark splitting of the levels $\left(\sim 15 \mathrm{~cm}^{-1}\right)$ is also observed for optical centers of other rare-earth ions in silicate glasses, studied by selective laser spectroscopy in [13]. Such optical centers in Er,Yb:BSP glass can be arbitrarily called cubic, bearing in mind the high average symmetry of the coordination polyhedra of the rare-earth ions. Thus for pump conditions up to $100 \mathrm{~W}$, mainly optical centers of cubic symmetry participate in lasing of Er,Yb:BSP glass, and it is specifically these centers that determine the shape of the continuous luminescence band, in particular the presence of two maxima near the wavelengths $1534.9 \mathrm{~nm}$ and $1541.6 \mathrm{~nm}$.

For optical centers 6-12 (Fig. 3b), which appear for pump powers above $200 \mathrm{~W}$, a more complex Stark splitting structure is typical. In particular, for optical center 8 , we can pick out three groups with wavenumbers $0-3,13-$ 27 , and $42-51 \mathrm{~cm}^{-1}$; for center 11 , we see $0-8,23-31$, and $53 \mathrm{~cm}^{-1}$ etc. Such a substantial difference in the positions and grouping of Stark components for the different optical centers 6-12 suggests differences in the symmetry of these centers.

The differences in the symmetry of the optical centers in Er,Yb:BSP glass, connected with disordering of the glass structure, lead to variation in their basic optical parameters and consequently to different conditions for obtaining 
degenerate emission for the different optical centers. This explains the participation in the lasing process for $\mathrm{Er}, \mathrm{Yb}$ :BSP glass of spectrally different optical centers as the excitation conditions change.

Conclusion. We have studied the features of the spectroscopic properties of Er,Yb:BSP glass connected with the presence of different optical centers, and the spectral characteristics of the output emission of a laser based on this glass. We have established that changing the excitation conditions for Er,Yb:BSP glass leads to a change in the spectral composition of the generated emission within the entire luminescence band. We have shown that such behavior is connected with participation in the lasing process of at least 12 ensembles of optical centers having differences in the structure of the immediate environment of the rare-earth ion. For a pump power up to $100 \mathrm{~W}$, five similar optical centers with cubic symmetry participate in the lasing, and in the laser emission spectrum we observe two groups of lines with wavelengths near the maxima of the luminescence spectra, $1535 \mathrm{~nm}$ and $1541 \mathrm{~nm}$. As the pump power is increased up to $200 \mathrm{~W}$, seven more ensembles of optical centers are involved in the lasing process, which leads to the appearance of lasing lines within practically the entire luminescence band.

We would like to thank G. P. Yablonskii, A. L. Gurskii (B. I. Stepanov Institute of Physics, National Academy of Sciences of Belarus) and V. A. Chernyavskii (Institute of Molecular and Atomic Physics, National Academy of Sciences of Belarus) for useful discussions.

\section{REFERENCES}

1. S. A. Zolotovskaya, K. V. Yumashev, N. V. Kuleshov, and A. V. Sandulenko, Appl. Opt., 44, No. 9, 1704-1708 (2005).

2. Z. Liu, Ch. Qi, Sh. Dai, Y. Jiang, and L. Hu, Opt. Mater., 21, $789-794$ (2003).

3. M. Fan-Zhen, S. Feng, Zh. Chao-Bo, D. Xin, Sh. Mei-Ru, and Zh. Guang-Yin, Chin. Phys. Lett., 20, No. 10, 1739-1740 (2003).

4. Yu. P. Chukova, Anti-Stokes Luminescence and New Possibilities for Its Application [in Russian], Sov. Radio, Moscow (1980).

5. G. I. Ryabstsev, T. V. Bezyazychnaya, V. V. Parastchuk, A. G. Ryabtsev, M. V. Bogdanovich, A. I. Yenzhyieuski, L. L. Teplyashin, V. V. Kuznetsova, I. P. Petrovich, A. S. Kraskovskii, and S. N. Titovez, Opt. Commun., 252, No. 4-6, 301-306 (2005).

6. E. Georgiou, O. Musset, J. P. Boquillon, B. Denker, and S. Sverchkov, Opt. Commun., 198, 147-153 (2001).

7. Y. E. Sverchkov, B. I. Denker, G. V. Maximova, V. V. Osiko, and S. E. Sverchkov, in: Solid-State Lasers III (G. J. Quarles, ed.), Proc. SPIE, 1627, 37 (1992).

8. V. P. Gapontsev, M. V. Zhabotinskii, V. B. Kravchenko, and Yu. P. Rudnitskii, Laser Phosphate Glasses [in Russian], Nauka, Moscow (1980), p. 352.

9. M. A. El'yashevich, Spectra of Rare Earths [in Russian], Gos. Izdat. Tekhn.-Teoret. Lit., Moscow (1953), p. 114.

10. T. T. Basiev, E. V. Zharikov, V. I. Zhekov, T. M. Murina, V. V. Osiko, A. M. Prokhorov, B. P. Starikov, M. I. Timomechkin, and I. A. Shcherbakov, Kvant. Élektron., 3, No. 7, 1471-1477 (1976).

11. J. A. Keningstein and J. E. Geusic, Phys. Rev., 136, No. 3A, A726-A728 (1964).

12. A. A. Kaminskii, Spectroscopy of Crystals [in Russian], Nauka, Moscow (1975), pp. 92-122.

13. O. K. Alimov, T. T. Basiev, and S. B. Mirov, Tr. Inst. Obshchei Fiziki, 9, 6-49 (1987). 